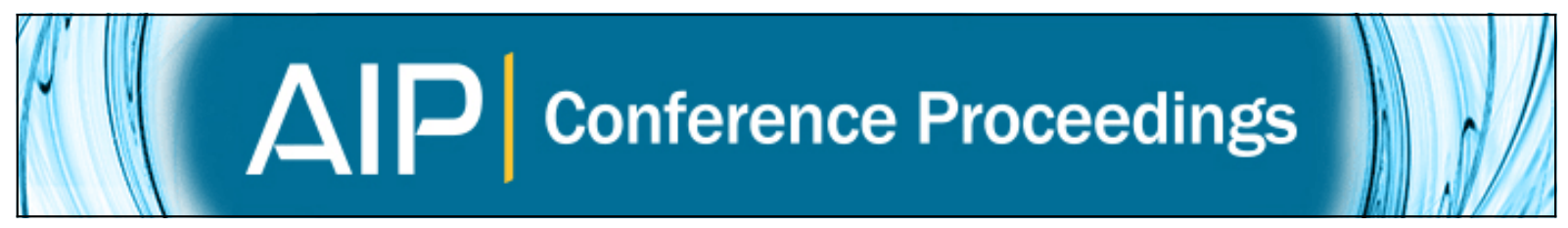

\title{
The most distant cosmological explosion
}

Poonam Chandra, Dale A. Frail, Derek Fox, S. R. Kulkarni, Edo Berger, S. Bradley Cenko, Douglas C.-J. Bock, Fiona Harrison, and Mansi Kasliwal

Citation: AIP Conference Proceedings 1381, 247 (2011); doi: 10.1063/1.3635836

View online: http://dx.doi.org/10.1063/1.3635836

View Table of Contents: http://scitation.aip.org/content/aip/proceeding/aipcp/1381?ver=pdfcov

Published by the AIP Publishing

\section{Articles you may be interested in}

The Origin of Subluminous O Stars

AIP Conf. Proc. 1314, 79 (2010); 10.1063/1.3536420

Explosive Nucleosynthesis in Luminous Hypernovae and Faint Supernovae

AIP Conf. Proc. 1294, 76 (2010); 10.1063/1.3518895

The Most Distant Galaxies: Theoretical Challenges

AIP Conf. Proc. 1294, 148 (2010); 10.1063/1.3518844

Explosive Nucleosynthesis in Supernovae and Hypernovae

AIP Conf. Proc. 1238, 9 (2010); 10.1063/1.3455981

Metal Enrichment of the IGM by Anisotropic AGN Outflows on Cosmological Scales

AIP Conf. Proc. 1201, 68 (2009); 10.1063/1.3293089 


\title{
The most distant cosmological explosion
}

\author{
Poonam Chandra*, Dale A. Frail ${ }^{\dagger}$, Derek Fox ${ }^{* *}$, S. R. Kulkarni ${ }^{\ddagger}$, Edo \\ Berger $^{\S}$, S. Bradley Cenko ${ }^{\mathbb{T}}$, Douglas C.-J. Bock ${ }^{\|}$, Fiona Harrison ${ }^{\ddagger}$ and \\ Mansi Kasliwal \\ ${ }^{*}$ Department of Physics, Royal Military College of Canada, Kingston, ON, Canada \\ ${ }^{\dagger}$ National Radio Astronomy Observatory, 1003 Lopezville Road, Socorro, NM 87801, USA \\ ** Dept. of Astronomy and Astrophysics, 525 Davey Laboratory, PSU, University Park, PA 16802 \\ $\doteqdot$ Department of Astronomy, California Institute of Technology, Pasadena, CA 91125 \\ ${ }^{\S}$ Harvard University, 60 Garden Street, Cambridge, MA 02138 \\ II Department of Astronomy, 601 Campbell Hall, University of California, Berkeley, CA 94720-3411 \\ "Combined Array for Research in Millimeter-wave Astronomy, P.O. Box 968, Big Pine, CA 93513
}

\begin{abstract}
At a redshift of 8.3 GRB 090423 marks the highest known redshift object in the Universe. By combining our radio measurements with existing X-ray and infrared observations, we estimated the kinetic energy of the afterglow, the geometry of the outflow and the density of the circumburst medium. Our best fit model is a quasi-spherical, high-energy explosion in a low, constant-density medium. We compare the properties of GRB 090423 with a sample of GRBs at moderate redshifts. We find that the high energy and afterglow properties of GRB 090423 are not sufficiently different from other GRBs to suggest a different kind of progenitor, such as a Population III star. However, we argue that it is not clear that the afterglow properties alone can provide convincing identification of Population III progenitors. We suggest that the millimeter and centimeter radio detections of GRB 090423 at early times contained emission from a reverse shock component. This has important implications for the detection of high redshift GRBs by the next generation of radio facilities.
\end{abstract}

Keywords: Cosmology, gamma-ray bursts, Late stages of stellar evolution, Interferometry

PACS: 98.80.-k, 98.70.Rz, 97.60.-s, 95.75.Kk

\section{INTRODUCTION}

Gamma-ray bursts (GRBs) are flashes of gamma-rays in the sky for just a fraction of a second to at most few minutes. In this small duration, they mark themselves as the most energetic cosmological explosions in the Universe after the Big Bang. Because of their extreme luminosities GRBs are detectable out to large distances by current missions. Due to their connection to core collapse $\mathrm{SNe}$ [41], they could in principal reveal the stars that form from the first dark matter halos $(z \sim 20-30)$ through to the epoch of reionization at $z=11 \pm 3$ and closer $[28,11,20,24]$. As bright continuum sources, occur at redshifts beyond those where quasars are expected, they could be used to study both the reionization history and metal enrichment of the early universe [39].

The fraction of detectable GRBs that lie at high redshift $(z>6)$ is, however, expected to be small $(<10 \%$; $[32,1])$. Until recently there were only two GRBs with measured redshifts $z>6$; GRB 050904 [25] and GRB 080913 [21] with $z=6.3$ and $z=6.7$, respectively. However, on April 23, 2009 the Swift Burst Alert Telescope (BAT) discovered GRB 090423 and the on-board X-ray Telescope (XRT) detected and localized a variable X-ray afterglow [37, 33]. In ground-based follow-up observations no optical 
counterpart was found but a fading afterglow was detected by several groups at wavelengths longward of $\mathrm{J}$ band $(1.2 \mu \mathrm{m})$.

We detected radio afterglow from the GRB using the Very Large Array ${ }^{1}$ (VLA). Broadband afterglow observations provide constraints on the explosion energetics, geometry, and immediate environs of the progenitor star. The afterglow has a predictable temporal and spectral evolution that depends on the kinetic energy and geometry of the shock, the density structure of the circumburst environment, and shock microphysical parameters which depend on the physics of particle acceleration and the circumburst magnetic field. In order to investigate the nature of the GRB 090423 explosion, we combine our radio measurements with published X-ray and NIR observations, and apply a model of the blast wave evolution to fit the afterglow data. We compare the explosion energetics, circumburst density, and other derived characteristics to a sample of wellstudied events, and discuss prospects for using afterglow measurements to investigate the nature of high- $z$ massive star progenitors.

\section{OBSERVATIONS}

We began observing a field centered at the NIR afterglow of GRB 090423 with the VLA about one day after the burst [9]. Our first detection of the GRB afterglow was not until about one week later at a flux density of $73.8 \pm 21.7 \mu \mathrm{Jy}$. We continued to monitor the GRB with the VLA until it faded below detection on day 64. Data reduction was carried out following standard practice in the AIPS software package. In order to improve our detection sensitivity, we averaged several adjacent observations. Datasets were combined in the $U V$-plane prior to imaging. By averaging three adjacent epochs (2009 May 1-May 3) when the afterglow was brightest, we estimate the best GRB position by fitting a 2-D Gaussian, which is; RA, Dec (J2000): $09^{h} 55^{m}(33.279 \pm 0.005)^{s}, 18^{d} 08^{\prime}(57.935 \pm 0.067)^{\prime \prime}$. This position is consistent with an earlier, less accurate WFCAM-UKIRT position from Tanvir et al. [38]. Table 1 gives the flux densities at the averaged epochs. For all epochs the flux density was measured at the position given above. We plot these data in Fig. 1. There is a broad plateau of about $45 \mu \mathrm{Jy}$ from 12 to 38 days, followed by a decline around day 55. The initial detections on days 8-10 could have contribution from a short-lived reverse shock.

We also observed GRB 090423 with the Combined Array for Research in Millimeterwave Astronomy (CARMA) at $95 \mathrm{GHz}$ band on 2009 Apr 25.19 UT. The observation was 8 hours in length. Data was obtained under non-ideal weather conditions. The peak flux at the VLA afterglow position is $450 \pm 180 \mu \mathrm{Jy}$. Castro-Tirado et al. [5] reported a secure millimeter band detection $(\lambda=3 \mathrm{~mm})$ at a flux density of $200 \mu \mathrm{Jy}$ with the Plateau de Bure Interferometer (PdBI) observed on 2009 Apr 23 \& 24.

Swift-XRT [4] observed the field of GRB 090423 for one week in Photon Counting (PC) mode. The XRT light curve is obtained from the on-line repository ${ }^{2}$ [13]. The X-ray

\footnotetext{
1 The Very Large Array is operated by the National Radio Astronomy Observatory, a facility of the National Science Foundation operated under cooperative agreement by Associated Universities, Inc.

2 http://www.Swift.ac.uk/xrt_curves
} 
TABLE 1. VLA 8.5 GHz flux densities of GRB 090423

\begin{tabular}{lll}
\hline \multicolumn{1}{c}{ Epochs combined } & Days since explosion & Flux density $(\mu \mathbf{J y})$ \\
\hline Apr 25.01-Apr 26.08 & $2.21 \pm 0.54$ & $50.9 \pm 30.9$ \\
May 01.05-May 03.98 & $9.34 \pm 1.64$ & $66.4 \pm 11.4$ \\
May 05.05-May 10.08 & $14.32 \pm 2.60$ & $43.7 \pm 8.9$ \\
May 12.99-May 15.05 & $20.71 \pm 1.06$ & $42.2 \pm 10.6$ \\
May 20.13-Jun 01.11 & $33.12 \pm 6.32$ & $49.6 \pm 11.0$ \\
Jun 20.00-Jun 26.91 & $62.00 \pm 4.33$ & $7.8 \pm 11.6$ \\
\hline
\end{tabular}

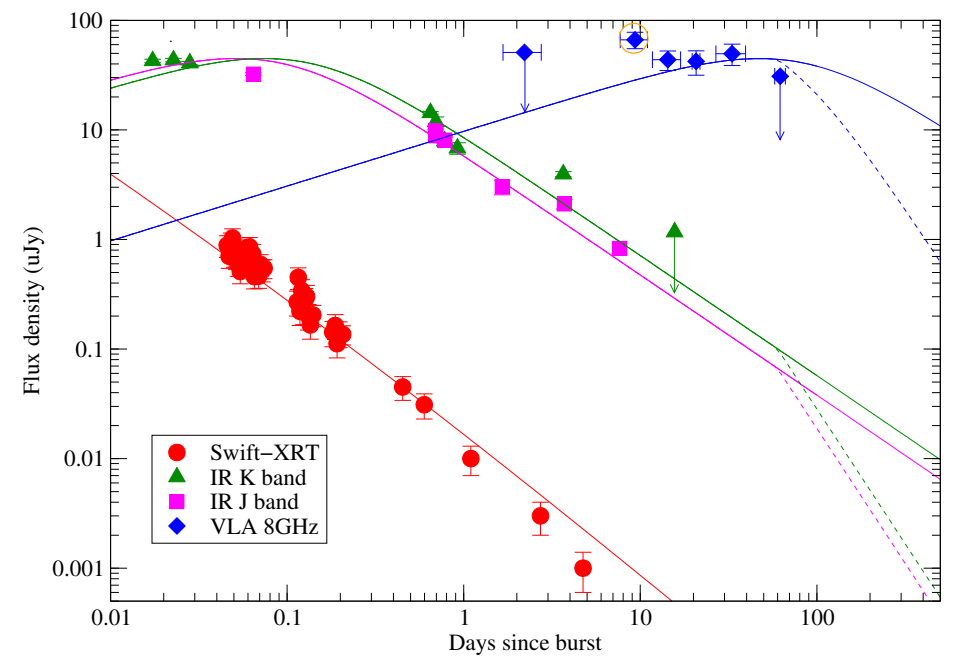

FIGURE 1. Multiwaveband observations for GRB 090423. The solid lines are best fit light curves for constant density isotropic model. The orange circled radio data likely has a contribution from RS. Dashed lines show model with a possible jet break around $t_{j}=45 \mathrm{~d}$.

spectrum is well-fit by a power-law model with a photon index $\Gamma=2.05_{-0.09}^{+0.14}$ and a total column density of $N_{H}=(8.7 \pm 2.5) \times 10^{20} \mathrm{~cm}^{-2}$ [37, 26]. We converted the $0.3-10.0$ $\mathrm{keV}$ counts to a flux density at $E=1.5 \mathrm{keV}\left(v_{0}=3.6 \times 10^{17} \mathrm{~Hz}\right)$ using the above value for $\Gamma$ and an unabsorbed count rate conversion of 1 count $=4.6 \times 10^{-11} \mathrm{erg} / \mathrm{cm}^{2} / \mathrm{s}$.

The NIR afterglow was observed by a variety of facilities worldwide; we have used values from Tanvir et al. [37]. To convert magnitudes to flux densities, we used zeropoint measurements from Fukugita et al. [18]. We have incorporated the Galactic extinction $(E(B-V)=0.029 ;[12])$ into these results. 


\section{RESULTS AND AFTERGLOW MODELLING}

Here we combine our radio data with the existing X-ray and NIR data and model the afterglow evolution, interpreting it in terms of the relativistic blast wave model [29]. In this model the afterglow physics is governed by the isotropic kinetic energy of the blast wave shock $E_{K \text {,iso }}$, the jet opening angle $\theta_{j}$, the density of the circumburst medium $n$, and the microscopic parameters such as electron energy index $p$, and the fraction of the shock energy density in relativistic electrons $\varepsilon_{e}$ and magnetic fields $\varepsilon_{B}$. The afterglow modeling software [42] assumes a standard synchrotron forward shock formulation. In the X-ray band, we exclude the data before $\sim 3900 \mathrm{~s}$, since it contains a flare which is more likely due to the GRB itself than the afterglow.

It is well known $[35,34,10]$ that the afterglow framework allows the above blast wave parameters to be constrained using multi-wavelength light curves. First, we note the constancy of the peak flux density $\left(F_{v, \max }\right)$ between the NIR and the radio bands in Fig. 1. If we interpret this as the passage of the synchrotron peak frequency $v_{m}$ through each band, this immediately rules out the wind model $\left(F_{v, \max } \propto t^{-1 / 2}\right)$ and favors a constant-density ambient medium $\left(F_{v, \max } \propto t^{0}\right)$. Another related constraint which comes from the Fig. 1 is the time of the peak in the NIR versus the radio bands. We note that in the NIR band, the light curve peaks at $\sim 0.08 \mathrm{~d}$. Thus if there was an early jet break the model predicts that the synchrotron peak frequency $v_{m}$ would evolve from NIR to radio band around day $10\left(v_{m} \propto t^{-2}\right)$, however, for the isotropic model $v_{m}$ should pass through the $8.5 \mathrm{GHz}$ band around $\sim 50$ days $\left(v_{m} \propto t^{-3 / 2}\right)$. Since radio light curve indeed peaks at about $50 \mathrm{~d}$, this confirms that the jet break has not occurred at least until the afterglow peaked in radio band.

Second, the declining part of the IR light curve is well fit by a power law with a decay index $\alpha=-1.10 \pm 0.27$. Whereas the overall X-ray light curve after 3900s is well fit with a power law index of $\alpha=-1.35 \pm 0.15$. For the isotropic, constant density model we expect the flux at a given frequency $v_{\text {obs }}$ to decline as $t^{3(1-p) / 4}$ for $v_{m}<v_{\text {obs }}<v_{c}$ and $t^{(2-3 p) / 4}$ for $v_{m}<v_{c}<v_{\mathrm{obs}}$, where $v_{c}$ is the synchrotron cooling frequency [35]. These relations give consistent values of $p$ for the NIR $(p=2.46 \pm 0.36)$ and X-ray $(p=2.46 \pm 0.20)$.

Additionally, using Eq. 4 of Freedman \& Waxman [16] and X-ray flux on day 1, we obtain the fireball electron energy per unit solid angle in an opening angle $1 / \Gamma$ on $t=1$ $\mathrm{d}$ to be $\varepsilon_{e} E / 4 \pi=7.4 \times 10^{51}$ ergs. If we assume $\varepsilon_{e}=1 / 3$ [16], then the total fireball energy per unit solid angle in this opening will be $E / 4 \pi=2.5 \times 10^{52}$ ergs.

We summarize our robust inferences based on this preliminary analysis: (a) the data favors an isotropic explosion in a constant density medium; (b) the cooling frequency lies between the IR and X-ray bands; (c) the afterglow kinetic energy is large. From Tanvir et al. [37] we also know that the extinction due to a putative host galaxy is negligible $\left(A_{V}<0.08\right)$.

We now move on to more detailed modeling [42] guided broadly by these preliminary results. We fit a constant density model for parameters: $E_{K \text {,iso }}, \theta_{j}, n, p, \varepsilon_{e}$ and $\varepsilon_{B}$. All parameters were allowed to vary freely except that we fixed $p=2.46$ to lie in a narrow range $( \pm 0.20)$. The best fit parameters are tabulated in Table 2. Our best fit model is plotted in Fig. 1. 
TABLE 2. Best fit parameters for multiwaveband modeling of GRB 090423 for $p=2.46 \pm 0.2$

\begin{tabular}{lll}
\hline Parameters & \multicolumn{1}{c}{ Isotropic } & \multicolumn{1}{c}{ Jet $\left(t_{j}>45 \mathbf{d}\right)$} \\
\hline$E_{\gamma}(\mathrm{ergs})$ & $1.0 \times 10^{53}$ & $>2.2 \times 10^{51}$ \\
$E_{K}(\mathrm{ergs})$ & $3.8_{-1.7}^{+9.8} \times 10^{53}$ & $>8.4_{-3.7}^{+21.6} \times 10^{51}$ \\
$n\left(\mathrm{~cm}^{-3}\right)$ & $0.90_{-0.06}^{+0.11}$ & $\ldots$ \\
$\varepsilon_{B}(\%)$ & $0.016_{-0.015}^{+0.024}$ & $\ldots$ \\
$\varepsilon_{e}$ & $0.28_{-0.01}^{+0.10}$ & $\ldots$ \\
\hline
\end{tabular}

This simple model provides a reasonable fit to the data. The model implies GRB kinetic energy to be $E_{K}=3.8 \times 10^{53} \mathrm{erg}$. However, the last measured data point is around day 65 (radio band) and the last detections in the radio and NIR bands are at about day 40 and day 46, respectively. Therefore a late jet break cannot be ruled out by these data. To illustrate this more concretely we overlay our best-fit model in Fig. 1 with a late jet break $t_{j} \sim 45 \mathrm{~d}$. The implied jet opening angle $\theta_{j}>0.21 \mathrm{rad}$ reduces both the radiated and the kinetic energies of this event by a factor of $\sim 45$. In this case the isotropic equivalent gamma-ray energy $\mathrm{E}_{\gamma}=1 \times 10^{53} \mathrm{erg}$ [40] and the blastwave kinetic energy $E_{K}=3.8_{-1.7}^{+9.8} \times 10^{53}$ erg give lower limits to the beaming-corrected values of $E_{\gamma}>2.2 \times 10^{51} \mathrm{erg}$ and $E_{K}>8.4_{-3.7}^{+21.6} \times 10^{51} \mathrm{erg}$, respectively.

The radio data point on day $9.34(t \sim 1 \mathrm{~d}$ in the rest frame) has high flux and does not go through the best fit forward shock model. Such early, short-lived radio emission is fairly common in GRBs at lower redshifts and is thought to be due to a contribution from afterglow reverse shock (RS) [27, 36, 30].

\section{DISCUSSION AND CONCLUSIONS}

GRB 090423 is the highest-redshift object for which we have multi-wavelength observations, including good quality radio measurements. Below we address the question as to what can we learn about properties of the explosion and environs for this highest-redshift GRB based on its afterglow properties? And, can we identify any differences between high and low redshift GRBs which indicate that they might arise from different progenitors? In particular, the initial generations of stars in the early universe are thought to be brighter, hotter and more massive $\left(>100 M_{\odot}\right)$ than stars today [22, 3]. Detecting these so-called Population III (Pop III) stars is one of the central observational challenges in modern cosmology, and the best prospect appears to be through observing their stellar death [23] via a supernovae ( $\mathrm{SNe}$ ) or gamma-ray burst explosion. It is worth asking what observational signatures could signal a Pop III GRB.

Other than GRB 090423, only one other $z>6$ event, GRB $050904(z=6.26)$, has high quality broadband afterglow measurements. In Fig. 2 we plot the best-fit parameters of these two GRBs along with a sample of well-studied lower redshift events from Panaitescu \& Kumar [31]. Both high redshift bursts stand out in terms of their large blast wave energy ( $>10^{52} \mathrm{erg}$ ). We know from samples of well-studied afterglows $[15,31,42]$, that most have radiative and kinetic energies of order $\sim 10^{51} \mathrm{erg}$. In the 

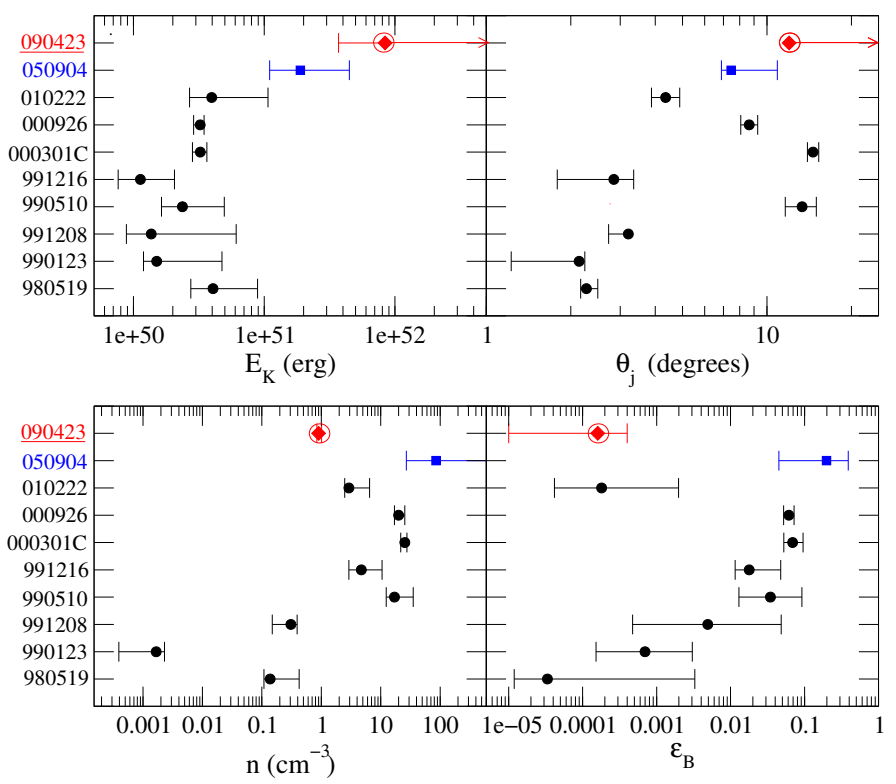

FIGURE 2. Comparison of GRB 090423 best fit parameters with few moderate- $z$ GRBs $(z \sim 1-3)$ from Panaitescu \& Kumar [31] and with the high- $z$ GRB $050904(z=6.295,[19,14])$. Here the upper limit on GRB $090423 E_{K}$ is $3.8_{-1.7}^{+9.8} \times 10^{53} \mathrm{erg}$.

collapsar model the jet kinetic energy from a Pop III GRB could be 10-100 times larger than a Population II (Pop II) event [17, 23]. However, an energetic explosion does not appear to be an exclusive property of high- $z$ GRBs. There is a small but growing population of bursts with energy $>10^{52} \mathrm{erg}$, termed 'hyper-energetic GRBs' [6], which includes moderate- $z$ events like GRB 070125 [8] and GRB 050820A [7].

Another potentially useful diagnostic is the density structure in the immediate environs of the progenitor star. The radio data is a sensitive in situ probe of the density because its emission samples the optically thick part of the synchrotron spectrum. The afterglows of GRB 090423 and GRB 050904 are best fit by a constant density medium and not one that is shaped by stellar mass loss [10]. However, many afterglows at all redshifts are best fit by a constant density medium (e.g. [42]). The density obtained for GRB 050904 was the highest seen $\left(n \approx 84-680 \mathrm{~cm}^{-3}\right)$ for any GRB to date, while GRB 090423 with $n=0.9 \mathrm{~cm}^{-3}$ does not stand out (Fig. 2), indicating these two high redshift bursts exploded in very different environments. A circumburst density of order unity is predicted for Pop III stars, since this density is limited by strong radiation pressure in the mini halo from which the star was formed [2]. This is not an unique property, since many local $\mathrm{SNe}$ explode in tenuous media, and so density constraints are not useful to signal Pop III explosions. 
For the other afterglow parameters $\left(p, \varepsilon_{e}, \varepsilon_{B}\right.$ and $\left.\theta_{j}\right)$ there are no published predictions for how they may differ between different progenitor models. Thus we turn to considering the prompt high-energy emission of GRB 090423.

Metallicity can also be an important discriminant. There is a critical metallicity $\left(Z>10^{-3.5} Z_{\odot}\right)$ below which high-mass Pop III stars dominate $[3,1]$. The contribution of Pop III stars to the co-moving star formation rate is expected to peak around $z=15$ but their redshift distribution exhibits a considerable spread to $z \sim 7$. Thus we might find high-redshift GRBs with Pop III progenitors in "pockets" of low metallicity. Salvaterra et al. [33] argue for a lower bound of $Z>0.04 Z_{\odot}$ based on their detection of excess soft X-ray absorption by metals along the line of sight, in comparison to the Milky Way column density predicted from HI $(21 \mathrm{~cm})$ measurements. We do not consider this a robust measurement as it is sensitive to a range of unaccounted-for systematic effects, including: spectral variability; spectral curvature; low-amplitude X-ray flares; and the presence of intervening (cosmological) absorption systems along the line of sight.

Summarizing the above discussion, we do not find that the individual properties of GRB 090423 are sufficiently dissimilar to other GRBs to warrant identifying it as anything other than a normal GRB. We lack robust predictions of well-defined afterglow signatures that could allow us to unambiguously identify a Pop III progenitor star from its afterglow properties alone. Significantly larger numbers of GRBs at high redshift with well-sampled afterglow light curves, high-resolution spectra, and host galaxy detections are needed to determine if high redshift GRB progenitors differ in a statistical sense from those at low redshift.

We note that, like GRB 050904, the GRB 090423 afterglow indicates the signature of reverse shock (RS) emission in the radio, as seen in the VLA and PdBI data. [24] have studied the expected RS emission at high redshift, and they find that the effects of time dilation almost compensate for frequency redshift, resulting in a near-constant observed peak frequency in the mm band $(v \sim 200 \mathrm{GHz})$ at a few hours post-event, and a flux at this frequency that is almost independent of redshift. Further, the mm band does not suffer significantly either from extinction (in contrast to the optical) or scintillation (in contrast to the radio). Therefore, detection of $\mathrm{mm}$ flux at a few hours post event should be a good method of indicating a high redshift explosion. ALMA, with its high sensitivity ( $\sim 75 \mu \mathrm{Jy}$ in $4 \mathrm{~min}$ ), will be a potential tool for selecting potential high- $z$ bursts that would be high priority for intense followup across the spectrum. This will hopefully greatly increase the rate at which high- $z$ events are identified.

Finally, our data does not rule out a late jet break at $t_{j}>45 \mathrm{~d}$, which, as discussed above, makes the total explosion energy uncertain. Extremely sensitive VLA observations would be required to distinguish between the isotropic versus jet model. For a $2 \mathrm{hr}$ integration in $8 \mathrm{GHz}$ band, the EVLA with its order of magnitude higher sensitivity can reach sensitivity up to $2.3 \mu \mathrm{Jy}$ which will be able to detect the GRB 090423 for 2 years or 6 months if the burst is isotropic or jet-like, respectively. EVLA will thus be able detect fainter events and follow events like GRB 050904 and GRB 090423 for a longer duration, therefore obtaining better density measurements, better estimates of outflow geometry and the total kinetic energy. 


\section{ACKNOWLEDGMENTS}

We thank Bob Dickman for his generous allocation of VLA time and Joan Wrobel and Mark Claussen for the timely scheduling of the observations. This work made use of data supplied by the UK Swift Science Data Centre at the University of Leicester. PC is supported by NSERC discovery grants held by Kristine Spekkens and Gregg Wade at the Royal Military College of Canada.

\section{REFERENCES}

1. Bromm, V. \& Loeb, A. 2006, ApJ, 642, 382

2. Bromm, V., Yoshida, N., \& Hernquist, L. 2003, ApJL, 596, L135

3. Bromm, V., Yoshida, N., Hernquist, L., \& McKee, C. F. 2009, Nature, 459, 49

4. Burrows, D. N., Hill, J. E., Nousek, J. A., et al. 2005, Space Science Reviews, 120, 165

5. Castro-Tirado, A. J., Bremer, M., Winters, J.-M., et al. 2009, GCN Circ., 9273, 1

6. Cenko, S. B., Frail, D. A., Harrison, F. A., et al. 2010, ApJ, 711, 641

7. Cenko, S. B., Kasliwal, M., Harrison, F. A., et al. 2006, ApJ, 652, 490

8. Chandra, P., Cenko, S. B., Frail, D. A., et al. 2008, ApJ, 683, 924

9. Chandra, P., Frail, D. A., \& Kulkarni, S. R. 2009, GCN Circ., 9249, 1

10. Chevalier, R. A. \& Li, Z.-Y. 1999, ApJL, 520, L29

11. Ciardi, B. \& Loeb, A. 2000, ApJ, 540, 687

12. Dickey, J. M. \& Lockman, F. J. 1990, ARA\&A, 28, 215

13. Evans, P. A., Beardmore, A. P., Page, K. L., et al. 2007, A\&A, 469, 379

14. Frail, D. A., Cameron, P. B., Kasliwal, M., et al. 2006, ApJL, 646, L99

15. Frail, D. A., Kulkarni, S. R., Sari, R., et al. 2001, ApJL, 562, L55

16. Freedman, D. L. \& Waxman, E. 2001, ApJ, 547, 922

17. Fryer, C. L., Woosley, S. E., \& Heger, A. 2001, ApJ, 550, 372

18. Fukugita, M., Shimasaku, K., \& Ichikawa, T. 1995, PASP, 107, 945

19. Gou, L.-J., Fox, D. B., \& Mészáros, P. 2007, ApJ, 668, 1083

20. Gou, L. J., Mészáros, P., Abel, T., \& Zhang, B. 2004, ApJ, 604, 508

21. Greiner, J., Krühler, T., Fynbo, J. P. U., et al. 2009, ApJ, 693, 1610

22. Haiman, Z. 2008, Astrophysics \& Space Science Library, Eds. H. Thronson, A. Tielens, M. Stiavelli, Springer (astro-ph/0809.3926)

23. Heger, A., Fryer, C. L., Woosley, S. E., et al. 2003, ApJ, 591, 288

24. Inoue, S., Omukai, K., \& Ciardi, B. 2007, MNRAS, 380, 1715

25. Kawai, N., Kosugi, G., Aoki, K., et al. 2006, Nature, 440, 184

26. Krimm, H. A., Pasquale, M. D., Perri, M., et al. 2009, GCN Rep., 211

27. Kulkarni, S. R., Frail, D. A., Sari, R., et al. 1999, ApJL, 522, L97

28. Lamb, D. Q. \& Reichart, D. E. 2000, ApJ, 536, 1

29. Meszaros, P. 2006, Reports of Progress in Physics, 69, 2259

30. Nakar, E. \& Piran, T. 2004, MNRAS, 353, 647

31. Panaitescu, A. \& Kumar, P. 2001, ApJ, 554, 667

32. Perley, D. A., Cenko, S. B., Bloom, J. S., et al. 2009, AJ, 138, 1690

33. Salvaterra, R., Della Valle, M., Campana, S., et al. 2009, Nature, 461, 1258

34. Sari, R., Piran, T., \& Halpern, J. P. 1999, ApJL, 519, L17

35. Sari, R., Piran, T., \& Narayan, R. 1998, ApJL, 497, L17+

36. Soderberg, A. M. \& Ramirez-Ruiz, E. 2003, MNRAS, 345, 854

37. Tanvir, N. R., Fox, D. B., Levan, A. J., et al. 2009, Nature, 461, 1254

38. Tanvir, N., Levan, A., Kerr, T., \& Wold, T. 2009, GCN Circ., 9202, 1

39. Totani, T., Kawai, N., Kosugi, G., et al. 2006, PASJ, 58, 485

40. von Kienlin, A. 2009, GCN Circ., 9251, 1

41. Woosley, S. E. \& Bloom, J. S. 2006, ARA\&A, 44, 507

42. Yost, S. A., Harrison, F. A., Sari, R., \& Frail, D. A. 2003, ApJ, 597, 459 